\title{
Contribution à l'étude des voies de pénétration de parasites fongiques des nectarines Monilia laxa (Ascomycète-Discomycète) et Rhizopus stolonifer (Zygomycète-Mucorale)
}

\author{
C. Nguyen-The ${ }^{1}$, R. Hugueney ${ }^{2}$ et M. Arnoux ${ }^{3}$ \\ 1 INRA, Station de technologie, Domaine Saint-Paul, 84140 Montfavet; \\ 2 Laboratoire de Botanique et Phytopathologie, Université Lyon 1, 43, bd du 11-Novembre 1918, 69622 Villeurbanne Cedex; \\ 3 INRA, Domaine de Gotheron, 26320 Saint-Marcel-les-Valence, France
}

(reçu le 9-8-1988, accepté le 18-12-1988)

\begin{abstract}
Résumé - Le comportement de conidies de $M$. laxa et de spores de $R$. stolonifer déposés à la surface de nectarine, non blessée, a été suivi en microscopie électronique à balayage. Les conidies de $M$. laxa germent abondamment à la surface des nectarines et une forte proportion des fruits contaminés sont infectés. Parfois des «figures de pénétration" d'hyphes mycéliens par des «microfissures cuticulaires» sont observées, bien que la plupart de ces microfissures ne semblent exercer aucune attraction vis-à-vis du mycélium de $M$. laxa. Aucune pénétration directe du champignon à travers la cuticule n'a été observée. Les spores de $R$. stolonifer ne peuvent germer à la surface des nectarines en l'absence de substrat nutritif. L'addition de jus de pêche dans l'inoculum permet un important développement du champignon, associé à l'infection du fruit. Une altération de l'épiderme du fruit est observée, en particulier les «microfissures cuticulaires» semblent s'élargir sous l'action du parasite.
\end{abstract}

Monilia laxa - Rhizopus stolonifer - nectarine, pêche - microscopie électronique à balayage

Summary - A contribution to the knowledge of the penetration mechanism of fungal pathogens on nectarine: Monilia laxa (Ascomycete-Discomycete) and Rhizopus stolonifer (Zygomycete-Mucorale). Conidies of M. laxa and spores of $R$. stolonifer were deposited onto the surface of uninjured nectarine fruit and observed under scanning electron microscopy. Conidies of $M$. laxa vigorously germinated and an important percentage of the contaminated fruits became infected. In some instances, mycelial hyphae obviously penetrated through some cuticular microcracks, although in most cases the fungus did not exhibit any attraction toward these microcracks. Direct penetration of M. laxa through intact cuticle was never observed.

Spores of $R$. stolonifer did not germinate on fruit surface without a nutrient support. The addition of peach juice stimulated growth of the fungus and led to the infection of the fruit. An important breakdown of the epidermis was observed and cuticular microcracks seemed to widen out.

peach - scanning electron microscopy - Monilia laxa - Rhizopus stolonifer - nectarine

\section{Introduction}

Monilia laxa (Aderh. \& Ruhl.) Honey et, dans une moindre mesure, Rhizopus stolonifer (Ehrenb. ex Fr.) Lind sont les principaux parasites en conservation des pêches et nectarines en Europe (Anderson, 1925; Bompeix et al., 1979; Byrde et Willetts, 1977). Leur impact économique demeure important malgré les traitements fongicides effectués en verger (Arnoux, 1986). De plus, bien que des traitements post-récoltes donnent des résultats intéressants (Ogawa et al., 1963; Wells et Bennett, 1976; Bompeix et al., 1979, Laville et Souty, 1982), ceux-ci sont interdits en France, et le contrôle de ces deux champignons parasites est difficile.

La contamination de blessures par des spores de $R$. stolonifer ou par des conidies de M. laxa, conduit à la pourriture du fruit, même si l'inoculation survient plusieurs semaines avant maturité 
(Corbin, 1962; Nguyen-The et Souty, 1985). La barrière épidermique semble donc être un facteur limitant de l'infection. En particulier, la cuticule joue un rôle important dans la défense des végétaux contre leurs parasites (Kolattukudy et Koller, 1983). Fogle et Faust (1975) ont mis en évidence l'existence de "microfissures" dans la cuticule des pêches et nectarines. Celles-ci pourraient servir de "porte d'entrée" à $M$. laxa lors d'infections sans blessure de l'épiderme, bien que Wade (1956), pour l'abricot, et Hall (1971), pour les pêches, accordent une importance déterrninante aux stomates ou aux lenticelles. Le comportement de $R$. stolonifer à la surface de pêches ou de nectarines demeure inconnu.

Le présent travail a pour but de définir les voies de pénétration les plus probables lors de contaminations de nectarines par $M$. laxa et $R$. stolonifer. Pour cela, des observations en microscopie électronique à balayage ont été effectuées, conjointement à des tests d'inoculation de fruits en laboratoire.

\section{Matériel et Méthodes}

Les fruits proviennent des vergers de I'INRA de Gotheron (Saint-Marcel-les-Valence, Drôme) ou des vergers du CTIFL de Balandran (Bellegarde, Gard). Récoltés à maturité commerciale, ils appartiennent aux cultivars suivants : “Crimson-Gold», «Spring-red», «Fantasia», "Royal-Giant» (Nectarines) et «Red-Top" (pêches).

Les isolats de $M$. laxa et $R$. stolonifer proviennent de cultures monospores isolées respectivement de fruit pourri et de rameau de pêcher. Les cultures sont maintenues sur milieu Potato Dextrose Agar-PDA (Biomérieux), $M$. laxa étant repiqué sur un milieu $V 8$ agar ou inoculé à une nectarine quelques jours avant l'expérimentation.

Les conidies de $M$. laxa sont prélevées sur des cultures de 6 jours (à $25^{\circ} \mathrm{C}$ et à la lumière du jour) sur milieu V8 agar ou sur des fruits infectés par $M$. laxa (56 jours à $25^{\circ} \mathrm{C}$ après inoculation). Les spores de $A$. stolonifer sont prélevées sur des cultures de 4 jours $\left(25^{\circ} \mathrm{C}\right)$ sur milieu PDA. Dans les deux cas, une suspension réalisée dans l'eau distillée stérile est étalonnée à l'aide d'un hématimètre de Malassez, puis ajustée à la concentration de $10^{6}$ spores $/ \mathrm{ml}$. Les truits sont contaminés par une goutte de suspension de spores $(20 \mu \mathrm{l})$, celle-ci étant additionnée de jus de pêche (1 volume de jus pour 20 volumes de suspension) lors des essais nécessitant une forte germination des spores de $R$. stolonifer.

Des spores de $R$. stolonifer en germination ont aussi été obtenues en étalant une suspension de spores sur une feuille de cellophane déposée à la surface d'une couche de PDA (4mm d'épaisseur) dans une boîte de Petri. Après $8 \mathrm{~h}$ à $30^{\circ} \mathrm{C}$, la feuille de cellophane est prélevée et les spores en germination sont récupérées dans de l'eau distillée stérile. Des fruits ont été contaminés avec cette suspension, de la façon décrite ci-dessus.

Le matériel destiné aux observations en microscopie électronique à balayage est observé à l'état conge- lé ou préparé par déshydratation au point critique lorsque l'observation doit être différée. La congélation est effectuée dans l'azote pâteux sous atmosphère d'argon, en utilisant l'appareil à congélation SP 2000 Emscope. La sublimation des cristaux de glace éventuellement formés est suivie en microscopie à balayage $\left(\mathrm{JEOL} 35^{\circ} \mathrm{C}\right)$ sous une tension de $4 \mathrm{kV}$. Le matériel est ensuite métallisé à l'or puis observé sous une tension de 15 à $20 \mathrm{kV}$. Les échantillons destinés à la déshydratation par le point critique sont fixés au glutaraldéhyde $(2 \%)$ dans un tampon cacodylate $\mathrm{pH} \mathrm{7,2}$ $0,2 \mathrm{M}$ et déshydratés dans l'acétone.

\section{Résultats}

Un nombre important de "microfissures» existent à la surface des nectarines (Fig. 1). Elles semblent organisées radialement autour des lenticelles, puis se ramifient pour former un réseau, particulièrement dense autour de la cicatrice stylaire. La plupart des fissures ont l'aspect de "craquelures" larges dont le fond présente des «structures d'étirement». Dans certains cas, des "crevasses" plus profondes et plus étroites sont observées (Fig. 2). Aucune différence notable n'a été observée entre les nectarines appartenant aux différents cultivars, pour environ 40 échantillons étudiés en microscopie électronique à balayage. Des structures similaires existent à la surface des pêches, dont la pilosité très développée gêne l'observation (Fig. 3).

Les conidies de $M$. laxa déposées en suspension aqueuse à la surface des nectarines tendent à s'accumuler dans les "microfissures" (Fig. 4). Une abondante germination des conidies et un important développement des hyphes mycéliens sont observés après $24 \mathrm{~h}$ en chambre humide (Fig. 5). La majorité des conidies se développe de façon anarchique, apparemment sans attraction en direction des "microfissures", cependant dans quelques cas des tubes germinatifs se dirigent vers l'intérieur des crevasses (Fig. 6). Parfois, au fond de craquelures apparemment peu profondes, l'extrémité du mycélium prend un aspect contourné en «peloton» (Fig. 7). Un pourcentage important (jusqu'à 100\%) des fruits inoculés est infecté par $M$. laxa après 2 ou 3 jours en chambre humide. Ceci montre que les phénomènes de pénétration décrits ci-dessus semblent bien conduire à l'infection du fruit. Certains fruits inoculés ne présentent aucun symptôme de pourriture après 8 jours en chambre humide et pourtant un important développement de mycélium et des "tentatives" de pénétration (Fig. 8) n'ayant pas conduit à l'infection peuvent être observés.

A l'inverse de ce que l'on observe avec les conidies de $M$. laxa, aucune spore de $R$. stolonifer ne germe à la surface des nectarines 

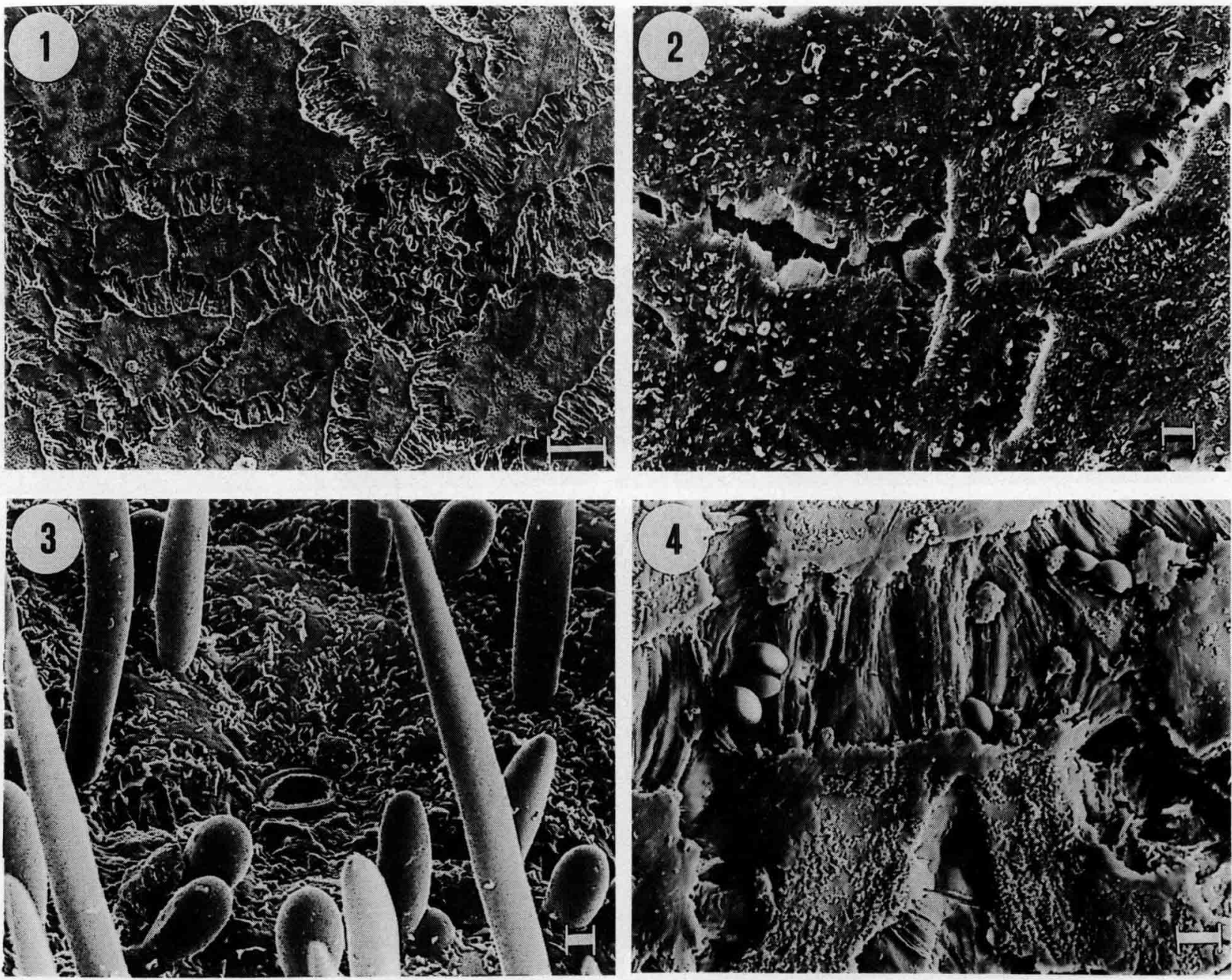

Fig. 1. Aspect de surface d'une nectarine montrant un réseau de microfissures cuticulaires dont le fond présente des structures d'étirement. $\mathrm{Cv}$ “Crimson-Gold». Echantillon observé congelé, ainsi que pour les autres figures, à l'exception des numéros $6,7,8$ où l'observation a été conduite après déshydratation au point critique. $-: 100 \mu \mathrm{m}$.

Fig. 2. Microfissure de type crevasse, étroite et d'aspect profond. Nectarine $C v$ «Crimson-Gold». - : $10 \mu \mathrm{m}$ dans les Figs 2 à 10.

Fig. 3. Aspect de surface d'une pêche montrant des microfissures cuticulaires similaires à celles observées sur les nectarines : Cv «Red-Top".

Fig 4. Conidies de $M$. laxa retenues dans une microfissure à la surface d'une nectarine : CV «Crimson-Gold».

(Fig. 9). De même, l'inoculation de fruits, sans blessure, par des spores de $R$. stolonifer ne permet pas d'obtenir une infection. En revanche, l'inoculation par des spores mises en germination sur milieu PDA puis reprises en suspension dans l'eau distillée, ou par des spores en suspension dans du jus de pêche dilué, conduit à l'infection du fruit en 24 à $48 \mathrm{~h}$. Une altération de l'épiderme localisée au niveau des microfissures est alors visible en microscopie électronique à balayage (Fig. 10). Le mycélium de $R$. stolonifer semble plus dense à l'intérieur des "microfissures» et celles-ci paraissent plus profondes et plus larges que sur un fruit sain.

\section{Discussion - Conclusion}

Bien que l'inoculation par $M$. laxa de nectarines non blessées conduise à l'infection, aucune pénétration "directe» n'a été observée contrairement, par exemple, à Botrytis cinerea sur fruit de tomate. Dans ce cas, le filament germinatif issu de conidies semble traverser la cuticule, indépendamment de toute discontinuité préexistante (Rijkenberg et al., 1980). A la surface des nectarines, les microfissures cuticulaires pourraient avoir un rôle mécanique en retenant les spores de champignon. De plus, quelques phénomènes de pénétration au niveau des microfissures ont été observés pour $M$. laxa., dans le cas de fissures profondes et peut-être non cicatrisées. Celles-ci seraient donc la principale voie de pénétration pour ce chamignon, rejoignant ainsi le cas de $B$. cinerea sur baies de raisin, où des craquelures cuticulaires péristomationues jouent un rôle prédominant dans l'infection (Bessis, 1972; Pucheu-Plante et Mercier, 1983). II en serait de même pour Phytophtora cactorum sur pomme (Mourichon et Bompeix, 1979). 

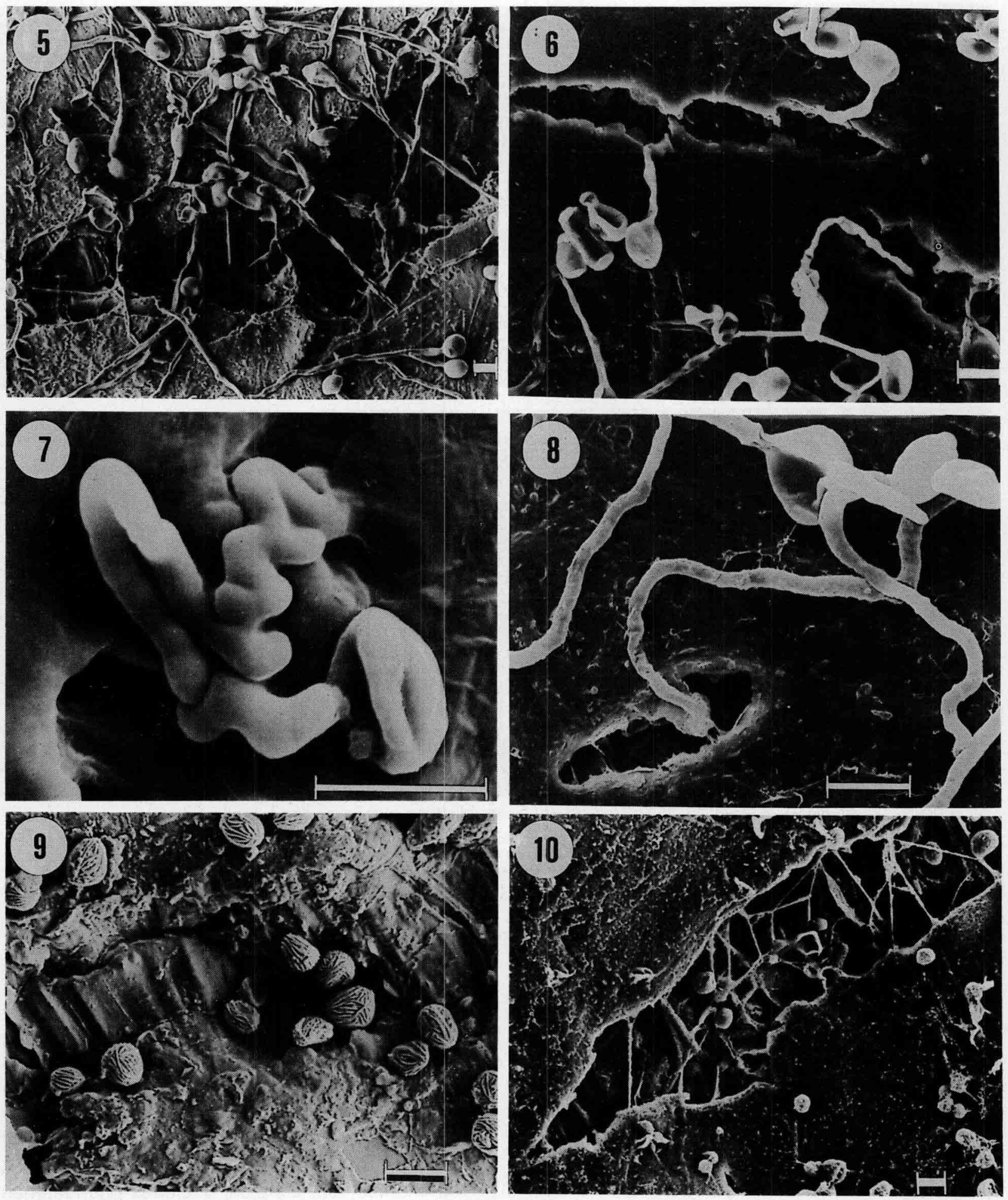

Fig. 5. Conidies de $M$. laxa à la surface d'une nectarine : CV “Fantasia». Observation effectuée après $24 \mathrm{~h}$ à $25^{\circ} \mathrm{C}$. Les conidies ont été déposées dans une goutte d'eau distillée. Les nombreux hyphes mycéliens semblent se développer de façon anarchique, indépendamment de la présence d'une crevasse cuticulaire.

Fig. 6. Pénétration de tubes germinatifs de $M$. laxa par une fissure d'aspect profond de nectarine : $\mathrm{Cv}$ "Crimson-Gold», (conditions expérimentales identiques à celles de la Fig. 5).

Fig. 7. Peloton de mycélium issu de condies de $M$. laxa au fond d'une microfissure d'aspect superficiel : Cv “Crimson-Gold» (conditions expérimentales identiques à celles de la Fig. 5).

Fig. 8. Tentative de pénétration d'un hyphe mycélien issu d'une conidie de $M$. laxa par une crevasse cuticulaire de nectarine $\mathrm{CV}$ «Royal-Giant», inoculé par une suspension de conidies dans l'eau distillée et observé après 8 jours à $25^{\circ} \mathrm{C}$ sans qu'il y ait eu infection du fruit.

Fig. 9. Spores de $R$. stolonifer à la surface d'une nectarine. Cv «Fantasia». Les spores ont été déposées en suspension dans l'eau distillée et observées après $24 \mathrm{~h}$ à $25^{\circ} \mathrm{C}$. Noter l'absence de germination.

Fig. 10. Aspect de surface d'une nectarine $24 \mathrm{~h}$ après inoculation par une suspension de spores de $R$. stolonifer additionnée de jus de pêche : Cv «Spring-Red». Les microfissures semblent colonisées et dégradées par le mycélium du champignon. 
L'absence de germination des spores de $R$. stolonifer au fond des microfissures peut s'expliquer par le fait qu'elles ne peuvent germer dans l'eau distillée (résultats non présentés), contrairement à $M$. laxa, dont les conidies germent sans apport nutritif externe. II y aurait donc peu (ou pas) de substances nutritives au fond des microfissures cuticulaires de nectarines, contrairement aux baies de raisin, où les craquelures péristomatiques présentent un exsudat important (Bessis, 1972), dont le rôle dans la germination des conidies de $B$. cinerea à la surface des baies semble bien établi (Doneche, 1986). Par contre, le mycélium de $R$. stolonifer, contrairement aux spores, semble se développer au fond des microfissures tout en les dégradant. II est possible que cette dégradation soit une hydrolyse des parois des cellules épidermiques sous l'action des enzymes pectinolytiques de $R$. stolonifer (Nguyen-The et Souty, 1985). Les microfissures cuticulaires seraient par conséquent des sites favorables à la pénétration du champignon, mais l'absence de substances permettant la germination des spores serait un facteur limitant.

La majorité des microfissures observées en microscopie électronique à balayage ne semblent pas favorables à la pénétration de $M$. laxa. Ceci, joint à l'observation de «tentatives» de pénétration n'ayant pas conduit à l'infection du fruit, suggère l'existence de mécanismes de résistance intervenant après la pénétration et limitant le rôle des «microfissures». Wade et Cruickshank (1973) ont mis en évidence des phénomènes de résistance de cette nature sur des abricots inoculés avant maturité.

Les «microfissures» cuticulaires constituent des points de faiblesse à la surface des nectarines, permettant la pénétration des parasites fongiques, bien qu'une certaine forme de cicatrisation limite l'infection dans la majorité des cas. La faible proportion de fissures permettant la pénétration des filaments germinatifs de $M$. laxa montre qu'il n'y a peut-être pas de relation entre le nombre de microfissures et la sensibilité d'un fruit. Fogle et Faust (1976) ont étudié la variabilité des microfissures cuticulaires parmi plusieurs clones de nectarines et de pêches. Cette variabilité pourrait permettre d'améliorer la résistance des nectarines à $M$. laxa, à condition de mieux connaître la structure des «microfissures» cuticulaires et les mécanismes de pénétration. En particulier, une étude de l'épiderme en microscopie électronique à transmission serait nécessaire. D'une façon générale, l'infection de fruits par un parasite fongique peut faire intervenir des enzymes cutinolytiques produites par le champignon - par exemple, Colletotrichum gloeospo- rioides sur papaye (Dickman et al., 1982) - ainsi que des substances antifongiques préexistantes à l'infection dans le fruit ou induites par le parasite - par exemple, la pomme vis-à-vis de Nectria galligena (Swinburne et Brown, 1975; Seng et al., 1985). Ces deux points devraient être vérifiés dans le cas des nectarines infectées par $M$. laxa ou $R$. stolonifer.

\section{Remerciements}

Ce travail a bénéficié d'une AIP de l'Institut national de la recherche agronomique.

\section{Références}

Anderson H.W. (1925) Rhizopus rot of peaches. Phy topathology 15, 122-124

Arnoux M. (1986) Qualité des pêches. Maîtrise des différents facteurs : interférence entre technique et socioéconomie. Arboric. fruit. 384, 46-49

Bessis R. (1972) Etude en microscopie électronique à balayage des rapports entre l'hôte et le parasite dans le cas de la pourriture grise. C.R. Acad. Sci. Paris Série $D$ 274, 2991-2994

Bompeix G. Coeffic M. \& Greffier P. (1979) Lutte contre les pourritures des pêches à Monilia spp., Botrytis sp. et Rhizopus sp. Fruits 34, 423-430

Byrde R.J.W. \& Willetts H.J. (1977) The Brown Rot Fungi of Fruit, their Biology and Control. Pergamon Press, Oxford

Corbin J.B. (1962) Factors determining the length of the incubation period of Monilia fructicola Honey in fruits of Prunus spp. Aust. J. Agric. Res. 14, 51-60

Dickman M.B., Patil S.S. \& Kolattukudy P.E. (1982) Purification and characterization of an extracellular cutinolytic enzyme from Colletotrichum gloeosporioïdes on Carica papaya. Physiol. Plant Pathol. 20, 333-347

Doneche B. (1986) La nature des exsudats de raisins et leur rôle dans la germination des conidies de Botrytis cinerea. Agronomie 6, 67-73

Fogle H.W. \& Faust M. (1975) Ultrastructure of nectarine fruit surface. J. Am. Soc. Hortic. Sci. 100, 74-77

Fogle H.W, \& Faust M. (1976) Fruit growth and cracking in nectarines. J. Am. Soc. Hortic. Sci. 101, 434438

Hall R. (1971) Pathogenicity of Monilia fructicola. Part II. Penetration of peach leaf and fruit. Phytopathol. Z. 72, 281-290

Kolattukudy P.E. \& Koller W. (1983) Fungal penetration of defensive barriers of plants, p. 79-100. In: Biochemical Plant Pathology (J.A. Callow, ed.) John Wiley \& Sons, Chichester

Laville E. \& Souty M. (1982) Aspects phytopathologiques de la qualité des pêches de la région sud de la France. Essais de traitements après récolte. Fruits 37 , 301-313

Mourichon X. \& Bompeix G. (1979) Mise en évidence des voies de pénétration du Phytophtora cactorum (L. et C.) Schroeter dans les pommes "Golden delicious". Etude réalisée en microscopie électronique à balayage. Fruits 34, 761-766 
Nguyen-The C. \& Souty M. (1985) Dégradation des substances pectiques de pêche par Rhizopus stolonifer. 1 - Rôle possible dans les interactions hôte-parasite. Sci. Aliments 5, 447-463

Ogawa J.M., Mathre J.H., Weber D.J. \& Lyda S.D. (1963) Effets of 2,6-Dichloro-4-Nitroaniline on Rhizopus species and its comparison with other fungicides on control of Rhizopus rot of peaches. Phytopathology 53, 950-955

Pucheu-Plante B. \& Mercier M. (1983) Etude ultrastructurale de l'interrelation hôte-parasite entre le raisin et le champignon Botrytis cinerea : exemple de la pourriture noble en Sauternais. Can. J. Bot. 61, 17851797

Rijkenberg F.H.J., De Leeuw G.T.N. \& Verhoeff K. (1980) Light and electron microscopy studies on the infection of tomato fruits by Botrytis cinerea. Can J. Bot. 58, 1394-1404
Seng J.M., Saindrenan P. \& Bompeix G. (1985) Induction of Nectria galligena mutants resistant to benzoic acid and study of their aggressiveness towards immature apples. J. gen. Microbiol. 131, 1863-1866

Swinburne T.R. \& Brown A.E. (1975) The biosynthesis of benzoic acid in Bramley's seedling apples infected by Nectria galligena Bres. Physiol. Plant Pathol. 6, 259-264

Wade G.C. (1956) Investigations on brown rot of apricots caused by Sclerotinia fructicola (Wint.) Rehm. F-The occurrence of latent infection in fruit. Aust. J. Agric. Res. 7, 504-515

Wade G.C. \& Cruickshank R. (1973) The development of suceptibility of stone fruit to brown rot during ripening. Abstr. Papers 2nd Int. Congr. Plant Pathol. 0006 Wells J.M. \& Bennett A.H. (1976) Hydrocooling and hydraircooling with fungicides for reduction of postharvest decay of peaches. Phytopathology 66, 801-805 